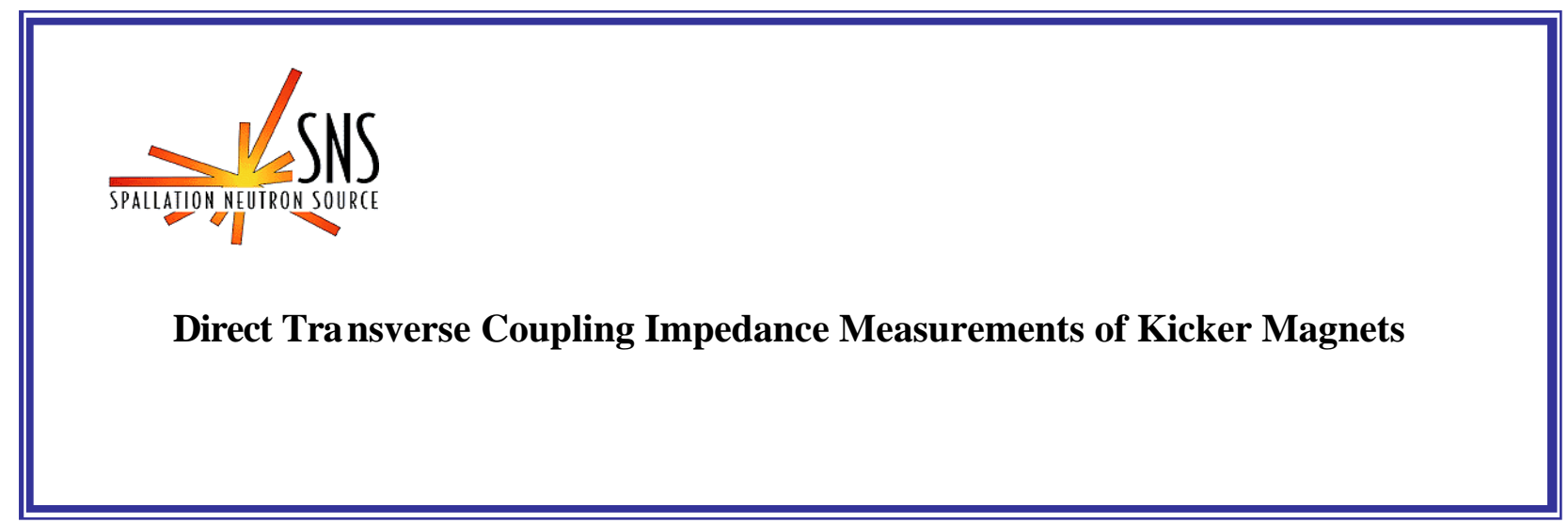

BNL/SNS TECHNICAL NOTE

NO. 120

H. Hahn

BNL, Upton, NY 11973, USA

March 20, 2003

COLLIDER-ACCELERATOR DEPARTMENT

BROOKHAVEN NATIONAL LABORATORY

UPTON, NEW YORK 11973 


\title{
Direct Transverse Coupling Impedance Measurements of Kicker Magnets
}

\author{
H. Hahn \\ Brookhaven National Laboratory, Upton, New York 11973
}

\section{Introduction}

Establishing and maintaining the coupling impedance budget is an important task during the design and construction phase of any accelerator or storage ring. The impedance of many components can be estimated using handbook expressions [1] or by analytical or numerical model computations [2, 3], but verification of the dominant contributions by measurement is common practice [4]. Typically, the transverse impedance contribution from kicker magnets is important, if not dominant, and has received the corresponding attention. The theoretical basis and a method of transverse impedance measurement involving a single loop was reported in the seminal paper by Nassibian and Sacherer [5]. The by now standard method was suggested by Caspers [6] and involves the insertion of twin-wires into the magnet aperture and the measurement of the forward transmission scattering coefficient with network analyzers. The twin-wire method was first applied by Walling et al. [7], and its interpretation was improved by the Vaccaro group [8]. Applying the wire method to frequencies at $\sim 1 \mathrm{MHz}$ and below entails difficulties and other methods must be considered. For example, the transverse impedance study of the LHC dump kicker was performed by Caspers et al. with multi-turn loops [9].

In the case of the SNS (Spallation Neutron Source) accumulator ring, the extraction kicker magnet represents the most critical item and was intensively studied using the twin-wire method with the procedure and results reported by Davino and Hahn (DH) in a recent paper [10]. The frequency range of interest to SNS covers low frequencies, from $\sim 50 \mathrm{MHz}$ down to below $1 \mathrm{MHz}$. Especially at the very-low frequency end, the signals become very weak and the results appear extremely noisy and their validity has been questioned. This provided the motivation to repeat the study and to search for measurement methods beyond the obvious averaging and smoothing of the data. In this paper a "direct" impedance measurement is suggested as complementary method.

The low-frequency range is on one hand the cause of the problem, but on the other hand it also presents the possibility of treating the magnet as lumped element. It was already pointed out by Nassibian and Sacherer, that the dominant coupling impedance is generated by the flux coupled to the external system. The DH model [10] for the coupled impedance consist of the magnet inductance with the external impedance in parallel, implying the possibility of a "direct" measurement of the components. The kicker magnet with its feed-through can be described by an equivalent circuit, the components of which can be measured separately, and which then serves for further impedance analysis. The mechanical construction of the kicker bus-bar provides a short gap which represents the appropriate location for the "direct" impedance measurement. 
The present paper describes a detailed comparison of results from the standard twinwire with those from the direct impedance measurement method. The gap impedance $Z^{G A P}$ corresponds to the $Z^{D U T}$ of the standard wire measurement and is interpreted as [5]

$$
Z_{\perp}=\frac{c}{\omega h^{2}} Z^{G A P}
$$

where the bus-bar aperture, $h$, replaces the wire spacing, $\Delta$. This relation was previously used at this laboratory by Blaskiewicz for qualitative estimates [11] and it was mentioned in the recent DH paper. The possibility of direct impedance measurement was also implied by the work of Blell [12]. However, the critical difference in the present approach is the use of the gap impedance rather than the impedance accessible at the external connector.

The direct impedance measurement is obviously less subject to noise and drift errors and this approach at obtaining the coupling impedance of a kicker magnet deserves consideration. The gap is only available during production and system testing and this method is not applicable to the fully installed kicker magnet. Furthermore, the gap impedance gives only the impedance coupled to the bus-bar and external circuitry. The uncoupled or "intrinsic" remains to be measured with the wire method. Fortunately, the "uncoupled" impedance contribution is at low frequencies small and can be ignored for the purpose of the impedance budget.

The detailed comparison presented here of results for the SNS extraction kicker from the standard twin-wire method with those from the direct impedance measurement confirm satisfactory agreement. In view of the significant systematic and random errors in the wire method, the results point to the direct gap impedance as a viable alternate transverse impedance measuring method.

\section{SNS Extraction Kicker}

All measurements were performed on the available full-size prototype for the fourteen SNS extraction kickers. A schematic view of a SNS window frame kicker magnet is shown in Fig. 1. The kicker dimensions are $h=24.8 \mathrm{~cm}, w=15.9 \mathrm{~cm}, l=36 \mathrm{~cm}$, the busbar has a gap of $g=2.6 \mathrm{~cm}$ and the two bus-bar plates are connected through side strips. The ferrite thickness is $t=2.54 \mathrm{~cm}$ with the ferrite type being CMD5005 (Ceramic Magnetics Inc., Fairfield, NJ, USA). The ferrite is the main source of resistive impedance at higher frequencies. Considering only the magnet part with uniform structure of bus-bar plates and ferrite frame leads to the estimate of a magnet inductance of $\sim 0.7 \mu \mathrm{H}$.

Capacitances within the kicker structure must be taken into account because they lead to a resonant behavior $[13,14]$. In the SNS kicker case, the main contribution to the capacitance is coming from the bus-bar end plates which provide the connection to the feed-thru, but the non-negligible permittivity of the ferrite can also make a contribution

coming from the bus-bar itself. Summing the various contributions leads to an estimated capacity of $C_{b b}=29 \mathrm{pF}$ for the full-size SNS prototype. Considering only the magnet part with uniform structure, and especially ignoring the capacitance of end plates and 
feed-thru, leads to the estimate for the capacitance of $\sim 10 \mathrm{pF}$. The uniform magnet section is estimated to have a characteristic impedance of $\sim 245 \Omega$ and a propagation velocity of $65 \%$ of light, or $<2 \mathrm{~ns}$ across the magnet. In the frequency range of interest, that is below $100 \mathrm{MHz}$, the magnet is indeed a lumped structure.

The kicker bus-bar is connected to the external pulsed power source via a coaxial feed-thru. Its inductance was measured to be $\sim 0.36 \mu \mathrm{H}$ and its capacity $\sim 11 \mathrm{pF}$. Installed, the feed-thru represents a capacitance of $\sim 31 \mathrm{pF}$. The center conductor of the feed-thru is stainless steel and a contributor to the resistive impedance at higher frequencies.
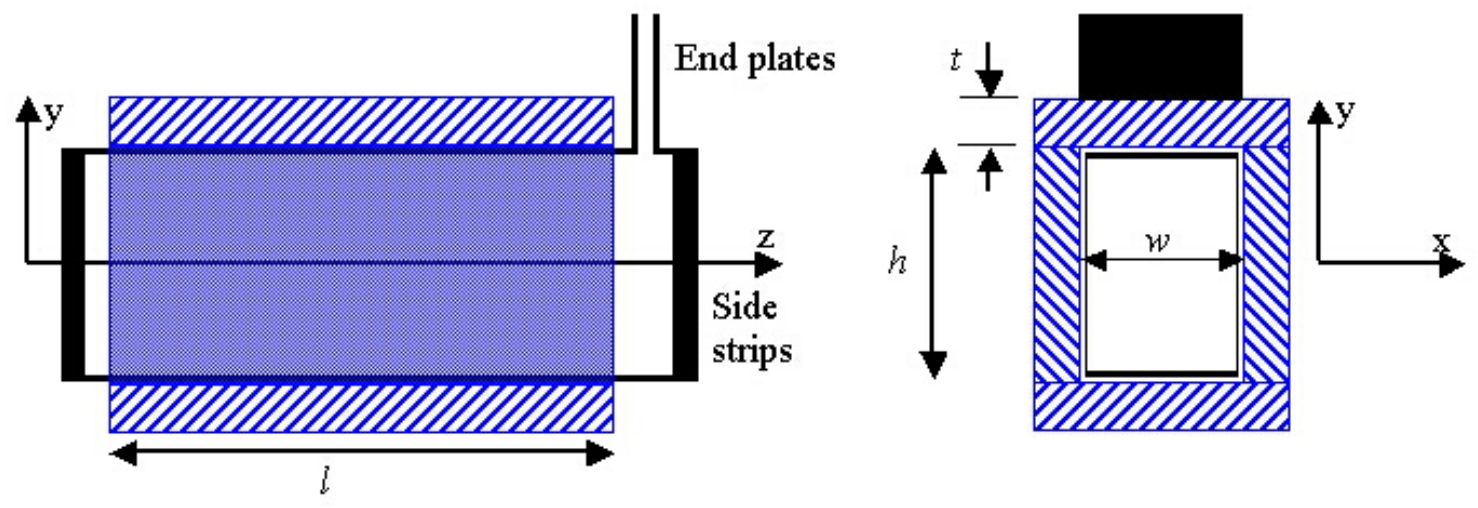

FIG. 1: Schematic view of a window frame kicker. Cross-hatched (blue) frames represent ferrite blocks. " $z$ " is the beam axis. $h$ is the aperture height, $w$ is the width, $l$ is the bus-bar length and $t$ is the ferrite thickness.

\section{Twin-wire Measurement Procedure}

The transverse coupling impedance of a kicker magnet can be measured on the bench by using the standard method in which a twin-wire (TW) "Lecher" line, simulating the beam, is inserted into the "Device Under Test". Using a network analyzer, the forward transmission coefficients $S_{21}^{D U T}$ of the kicker is determined and compared with the $S_{21}^{R E F}$ obtained in a reference measurement. The reference for the measurement of the uncoupled, here referred to as "intrinsic" impedance, is the free space as recommended by Caspers [4], rather than the beam tube The reference set-up for the measurement of the coupled impedance part is best obtained by shorting the magnet at the gap. Errors due to mechanical changes or drift in the network analyzer are thus eliminated or reduced.

The scattering coefficients were measured with the network analyzer, Agilent 8753ES, set for a linear frequency range from $30 \mathrm{kHz}$ to $70 \mathrm{MHz}$, with 801 points. The preeminent, low-frequency instrument errors of noise and drift were reduced by using a $30 \mathrm{~Hz}$ IF-bandwidth and by limiting the time between measuring the DUT and the reference to minutes. All critical measurements were obtained by averaging three sweeps. With exception of the "open" magnet, the date is shown with a $1 \%$ smoothing applied after averaging. 
The twin-wire measurements were performed by using a TW-line, home-made from $5 \times 7.5 \mathrm{~mm}$ rectangular tubes, $120 \mathrm{~cm}$ long, and adjusted for different spacing, as shown in Fig. 2. For calibration purposes, TW-lines were compared with a commercial line CQ 551 which has a spacing of $d=2 \mathrm{~cm}$ and a characteristic impedance of $450 \Omega$. The characteristic impedance of the TW-lines are obtained from a reference measurements with the communication signal analyzer, Tektronix CSA 803 and can be scaled according to the expression for round wires [15],

$$
Z_{T W}=\frac{c \mu_{0}}{\pi} \operatorname{acosh}(\mathrm{d} / \mathrm{b}) \sim 86.5 \Omega \cdot \operatorname{acosh}(\mathrm{d} / \mathrm{b})
$$

The proportionality factor of 86.5 , instead of the nominal $120 \Omega$, accommodates the rectangular lines. The TW lines with $d=28.6,40.6,53.6 \mathrm{~mm}$ will be identified according to their impedance as TW210, TW240, TW 265.

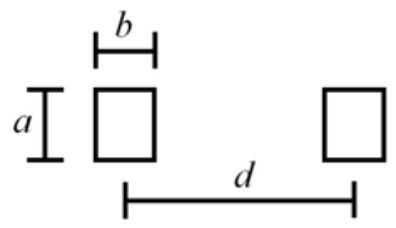

FIG. 2: The homemade twin-wire (TW) line. The dimensions are: $a=7.5 \mathrm{~mm}, b=5 \mathrm{~mm}$, and $d=28.6 ; 40.6 ; 53.4 \mathrm{~mm}$.

The TW lines are connected to the $50 \Omega$ cables of the network analyzer by means of commercial (North Hills) wide-band transformers with a center-tapped secondary winding, serving as $180^{\circ}$ hybrid. Transformers with different secondary impedance values and bandwidth were used and are identified according to their impedance as

X450 (NH15889 with $100 \mathrm{kHz}-65 \mathrm{MHz}$ ),

X300 (NH15880 with $100 \mathrm{kHz}-100 \mathrm{MHz}$ ), X200 (NH15888 with $10 \mathrm{kHz}-60 \mathrm{MHz}$ ).

TW-lines and transformers were combined in different combinations without resistive impedance matching. This departure from the standard treatment is justified because at the $\sim 1 \mathrm{MHz}$ frequency end, which was the primary focus of the study, the concept of transmission line impedance is immaterial: the wires of the TW-line only provide the connections between magnet and the transformers. The experimental calibration measurements discussed in the sequel confirmed this approach.

The forward scattering coefficient for a lumped series impedance, $Z$, between two transmission lines with characteristic impedance, $R_{c}$, and each of length, $l$, is derived in the Appendix leading to the low-frequency, low impedance approximation

$$
\frac{S_{21}^{D U T}}{S_{21}^{R E F}} \approx \frac{2 R_{0}}{Z+2 R_{0}}+j 2 k l \frac{Z}{R_{C}} \frac{R_{C}^{2}-R_{0}^{2}}{R_{0}^{2}}
$$

where $R_{0}$ is the instrument impedance. The effect of a mismatch is small, nevertheless, in order to assure a smooth transition to the upper frequency end, it would in general be prudent to match line and transformer impedance. 
The scattering coefficients are interpreted according to the HP-formula for lumped elements [16],

$$
Z^{D U T}=2 Z_{X}\left(1-S_{21}^{D U T} / S_{21}^{R E F}\right) /\left(S_{21}^{D U T} / S_{21}^{R E F}\right),
$$

with $Z_{X}$ the nominal secondary impedance of the transformer. The instrument accepts only a real reference value, but it is noted that the imaginary part of $Z_{X}$ impacts the results. The transverse coupling impedance now follows from the standard twin-wire measurement expression, as

$$
Z_{\perp}=\frac{c}{\omega \Delta^{2}} Z^{D U T}
$$

Note that only measurements in vertical, that is the kick direction, were made and $Z_{\perp}$ and $Z_{y}$ are not distinguished. For the typical case in kicker magnets, one has $Z^{D U T} \ll Z_{X}$, which leads to simplified approximations for the transverse coupling impedance of the DUT,

$$
Z_{\perp}=\frac{c Z^{D U T}}{\omega \Delta^{2}} \approx 2 \frac{c Z_{X}}{\omega \Delta^{2}}\left(1-S_{21}^{D U T} / S_{21}^{R E F}\right) \approx-2 \frac{c Z_{X}}{\omega \Delta^{2}} \ln \frac{S_{21}^{D U T}}{S_{21}^{R E F}}
$$

suggesting the use of the simpler log-formula [7]. The results presented in this paper were all obtained by using the conversion in the network analyzer corresponding to the lumped formula.

The transverse impedance result depends quadratically on the wire spacing, $\Delta$. It is recommended [4] to keep the wire spacing smaller than the half height of the magnet aperture in order to remain in the perturbation regime, $d \ll 12.25 \mathrm{~cm}$. In the typical case of the wire size much smaller than the wire spacing, the effective $\Delta$ is given by the center-to-center distance of the wires. Applying the results for round wires, one finds the effective spacing to be

$$
\Delta=d \sqrt{1-(b / d)^{2}} .
$$

Measuring the peak impedance of the "open", i.e. un-terminated magnet, employing different TW-lines gave results which are consistent with the use of the simpler $\Delta \approx d$. Clearly, the signal increases with the wire spacing and the signal to noise ratio is reduced. The definite measurements over the entire frequency range were thus made with the TW265 \& X300 combination, whereas other pairings were used mainly to establish parameter dependence.

\section{Calibrations}

In view of establishing confidence into the implications of the use of transformers with the twin-wire lines, the transformers and several TW combinations were tested. The input impedance on the secondary side of the transformer with the primary terminated in $50 \Omega$ is shown in Fig.3. The transformer ratio is frequency dependent and different for real and imaginary impedance, however the absolute value is relative constant. 

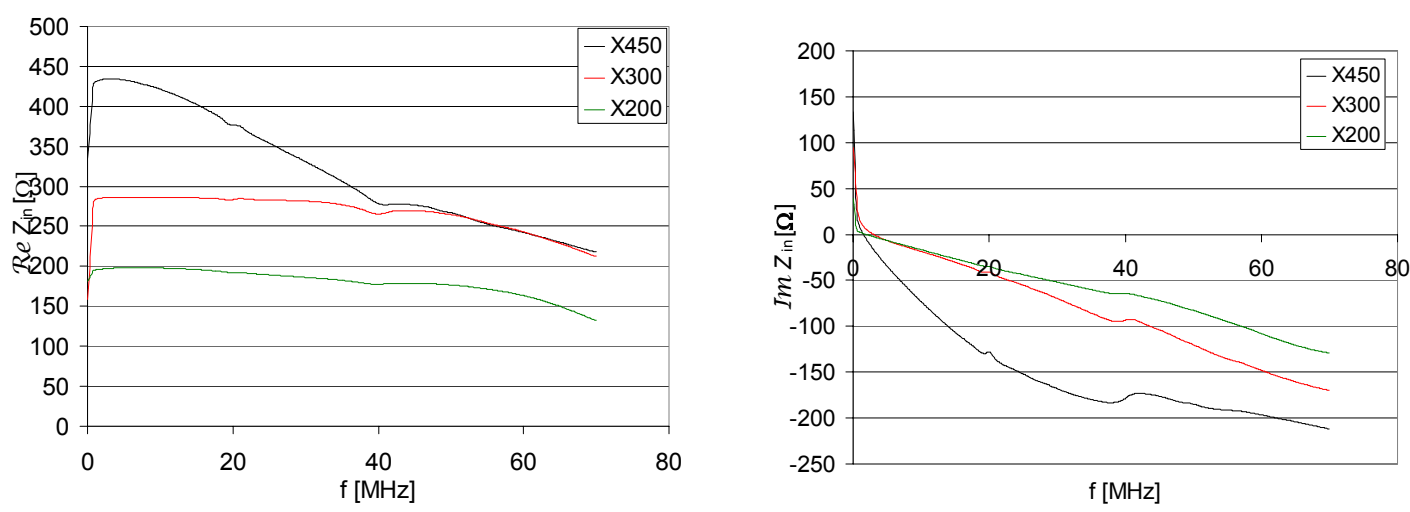

FIG. 3. Input impedance of transformer with $50 \Omega$ termination.

In order to determine the impact of the transformer ratio frequency dependence, a $Z_{p}=10 \mathrm{k} \Omega$ and $3.3 \mathrm{k} \Omega$ resistor was placed between the two wires of the TW265 line at the center between the X300 transformers and the resulting $Z^{D U T}$ is shown in Fig. 4. The expected values are $Z^{D U T}=Z_{X}^{2} / Z_{p}=9$ and $27.3 \Omega$ respectively. The agreement for the resistive component is reasonable in the low frequency range. The reactive part is unexpected and is attributed to the imaginary $Z_{X}$. Small contributions stem from the resistor leads or the mismatch of characteristic and instrument impedance.
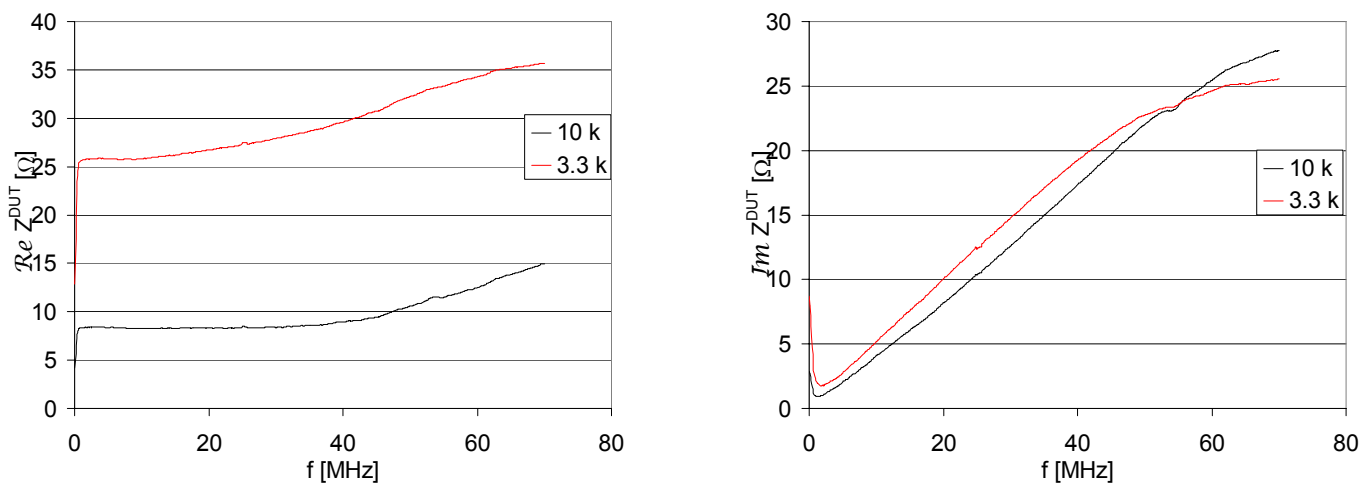

FIG.4. Calibration of the X300 transformer with the TW265 line.

The validity of the lumped element treatment and the use of $Z_{X}$ instead of the characteristic line impedance, $R_{C}$, in Eq. 4 was demonstrated by measuring the coupled impedance of the open SNS kicker. The TW lines were connected directly to the transformers without resistive matching. The measured resistive impedance of the $Z^{D U T}$ for the open kicker is shown in Fig. 5. The reference impedance to be used in the network analyzer was set to the nominal output transformer impedance $Z_{0} \rightarrow Z_{X}$. The results show that $Z^{D U T}$ is given by transformer impedance rather than the characteristic twin-wire impedance. Furthermore it is clear that the largest signal, and by implication, the most accurate results are obtained with the widest spacing of the TW265 line. 


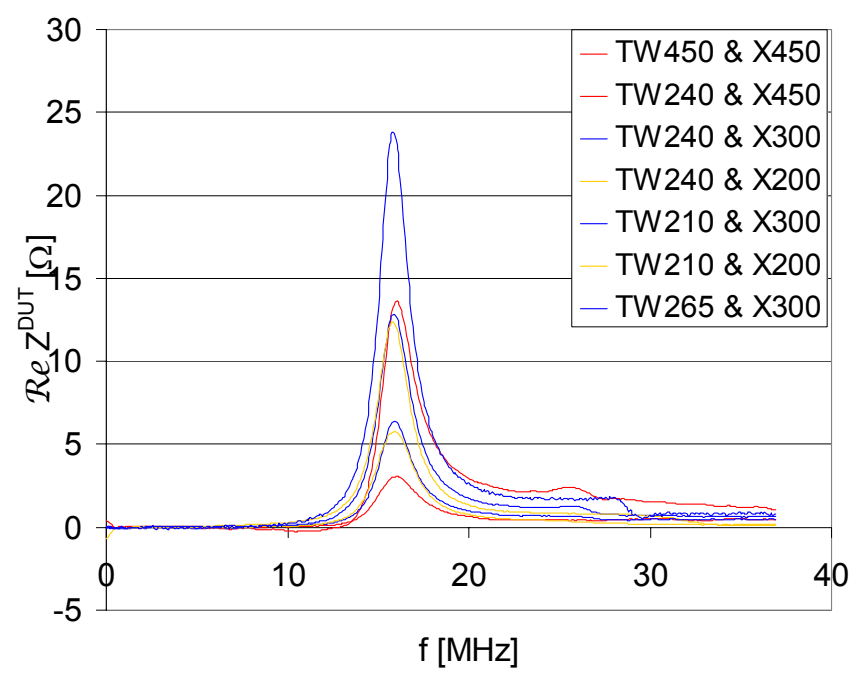

Fig. 5. Kicker open resonance measured with different combinations of twin wire and transformer.

At the same time, the use of the geometric wire spacing of the home-made TW lines for the effective spacing, $\Delta$, was confirmed. The wire spacing of the commercial TW450 line is taken as $\Delta_{450}=20 \mathrm{~mm}$ with sufficient accuracy to serve as basis for scaling of the home made twin-wires. The scaled effective spacing is thus found to be for

- TW265: $\Delta_{265} \approx \Delta_{450} \sqrt{23.8 / 3.06}=55.8 \mathrm{~mm}$

- TW240: $\Delta_{240} \approx \Delta_{450} \sqrt{13.0 / 3.06}=41.2 \mathrm{~mm}$

- TW210: $\Delta_{210} \approx \Delta_{450} \sqrt{6.02 / 3.06}=28.1 \mathrm{~mm}$

which is in good agreement with the geometric dimensions. The geometric spacing is used in the calculation of the transverse impedances. The above results indicate that the largest spacing line gives adequate signal strength for accurate results and the TW265 has been used together with the X300 transformer for most of the measurements.

\section{Intrinsic coupling impedance}

It was pointed out above that the coupling impedance of the kicker magnet consists of two contributions, the intrinsic impedance obtained with the bus-bar shorted, and the impedance coupled to the external system. Measurement of the intrinsic impedance must be made with regard to the reference value and involves necessarily a mechanical transfer of the twin-wire from reference to the device-under-test, a potential source of errors. Since, however, the value of the intrinsic impedance is small compared to the coupled impedance, the resulting errors are tolerable.

The measured wire impedance, that is the $Z^{D U T}$ for the magnet with shorted bus-bar together with the result for the open bus-bar is shown in Fig. 6. The data was taken with the X300 \& TW240 combination and both were taken with regard to the external reference. Also shown is the coupled impedance, which is measured with regard to the 
shorted bus-bar, now serving as in-situ reference. The results were obtained within less than 15 minutes and thus are able to confirm the assumption that the coupled impedance equals the difference between the total impedance minus the intrinsic impedance.
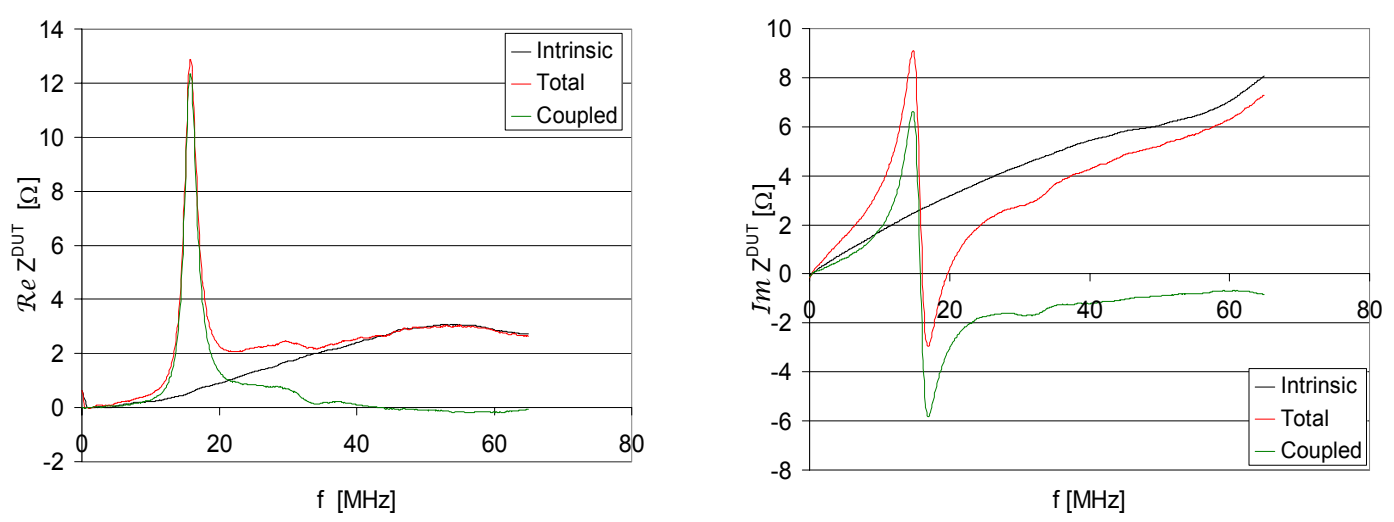

FIG. 6. The total impedance as sum of intrinsic and coupled contribution

The transverse intrinsic impedance, $Z_{y}$, which includes the contributions from shielding tubes and shorted eddy current strips, follows from the wire measurements of the shorted bus-bar by applying Eq. (5). The results, averaged over several transformer and TW line combinations, is shown in Fig.7. The intrinsic impedance can be represented as an essentially constant inductance, $L \approx 0.022 \mu \mathrm{H}$, with losses increasing with frequency, presumably due to the ferrite.

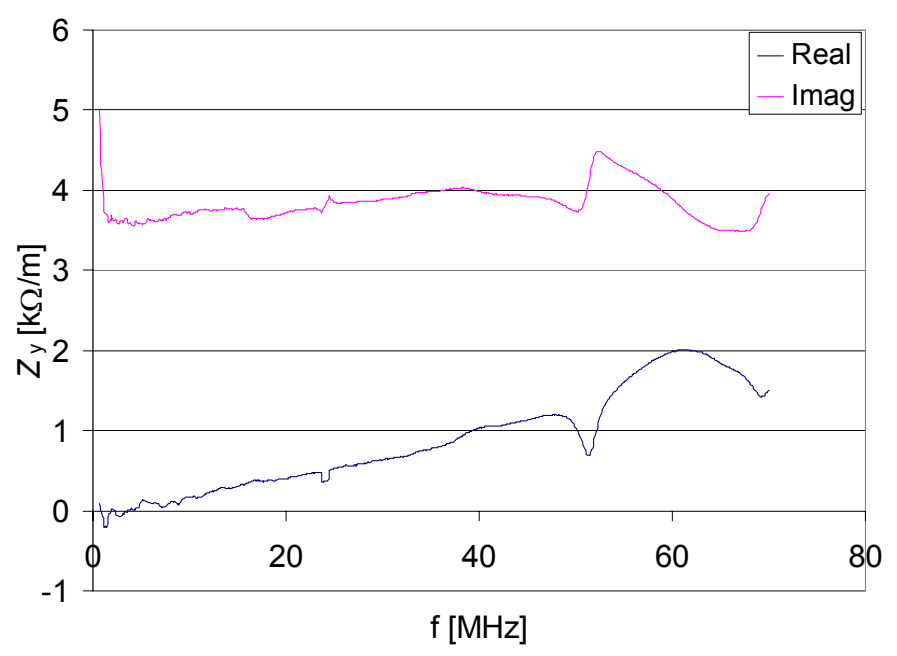

FIG.7. Intrinsic coupling impedance with the bus-bar shorted

\section{Comparison of the $Z^{D U T}$ and $Z^{G A P}$ Impedances}

The coupled part of the transverse coupling impedance can be obtained from the wire measurement $Z^{D U T}$ or, as proposed in this paper, from the direct impedance measurement at the gap $Z^{G A P}$. The SNS extraction kicker magnet was measured with feed-thru, reflecting the operational condition, and without feed-thru to establish a comparison with 
the DH paper [10]. Analyzing both cases leads to a better understanding and provides the basis for scaling to different geometries and terminations.

The wire impedances are measured versus the in-situ calibration obtained by shorting the bus-bar gap. Since there is no change of the measuring arrangement and the data are taken within minutes, the results are less noisy and drift is practically eliminated. The results reported in this section were performed with the X300 \& TW265 combination, which gives the strongest signal. Although the measurements were done at frequencies down to $30 \mathrm{kHz}$, the signals at frequencies below $\sim 1 \mathrm{MHz}$ are small and the conversion from $Z^{D U T}$ to $Z_{\perp}$ amplifies the random errors. In order to extend the lower frequency range, all curves were obtained by averaging over 3 cycles and a subsequent $1 \%$ smoothing was applied. The $1 \%$ smoothing reduces the open resonance peak by $\sim 4 \%$ but extends the credible frequency range to lower values, $\sim 0.7 \mathrm{MHz}$, which is below the $\sim 1 \mathrm{MHz}$ circulating frequency of the SNS beam.

The gap impedance $Z^{G A P}$ is measured directly with the network analyzer and the only errors are due to the clip lead inductance, $\sim 0.065 \mu \mathrm{H}$ and connector capacitance, $\sim 10 \mathrm{pF}$, which, however, are not negligible and must be considered.

\section{Magnet with open and shorted terminals}

The impedances for the magnet with feed-thru obtained from wire and direct measurements are compared in Fig.8. for the "open" and "shorted" terminations. Also shown are the "open" impedances for the magnet without feed-thru. Obviously, the "shorted" impedance without feed-thru does not exist. The wire measurements are scaled to the magnet aperture according to $(h / \Delta)^{2}$ in order to allow the comparison with the direct impedances. The differences between wire and direct impedances are mostly a shift of the resonance and can be explained, at least in part, by the clip lead inductance and connector capacity of the direct measurement cable.
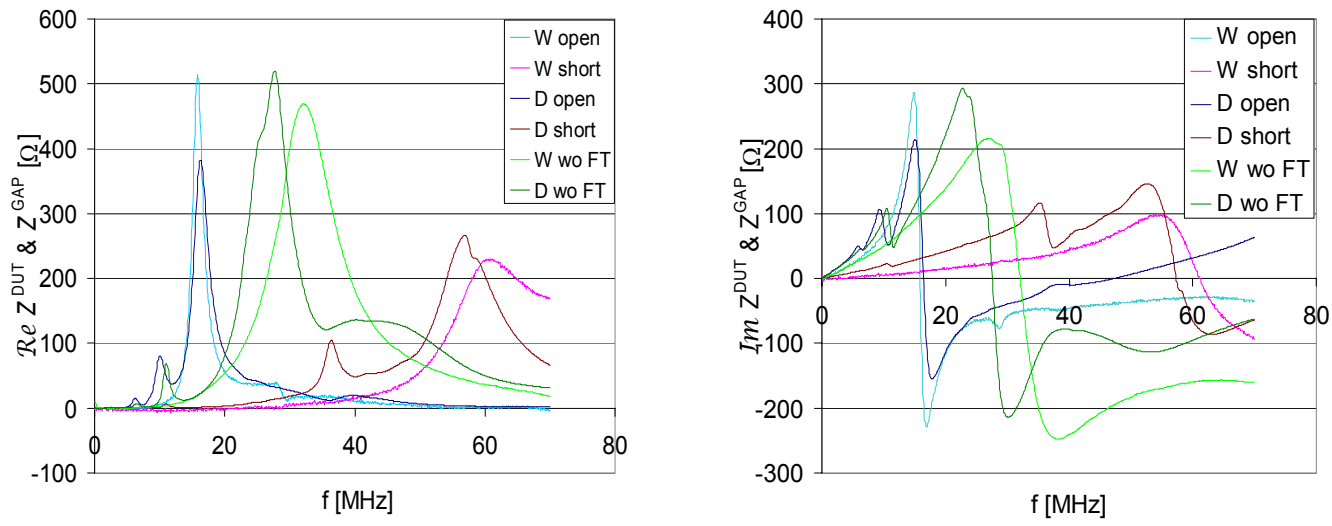

Fig. 8. Comparison of impedance from wire and direct measurement 


\section{Magnet impedance at terminal}

Direct measurement of the feed-thru impedance at the terminal with the gap open and shorted is shown in Fig. 9. The resonance with the gap open involves the magnet whereas the resonance with the gap shorted depends on the feed-thru only. No corresponding wire measurements exist for these results.
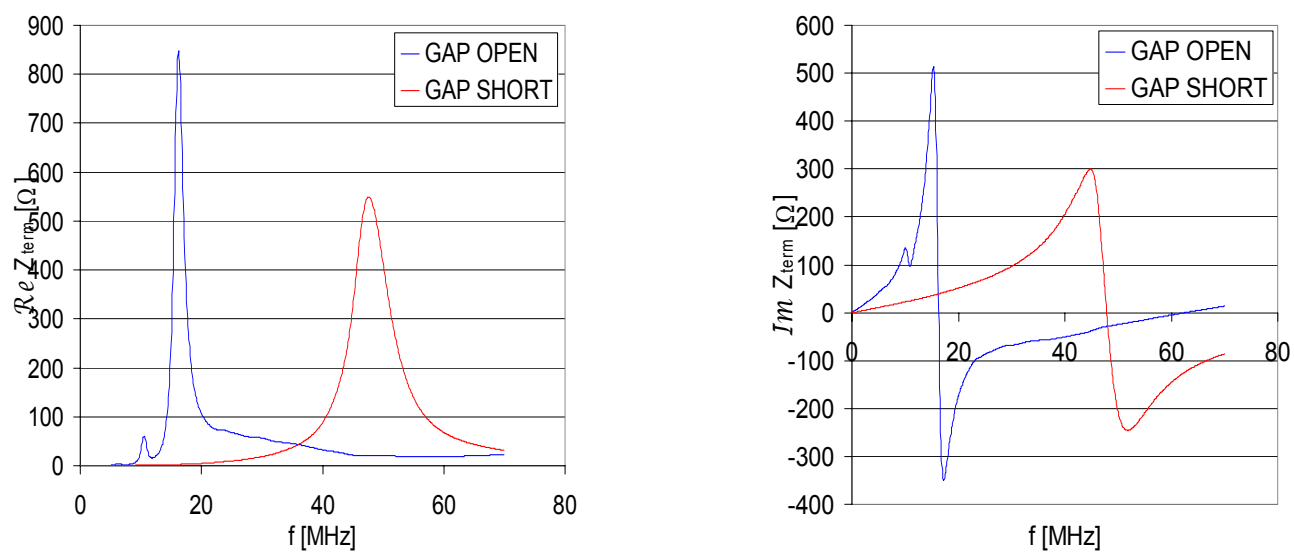

Fig. 9. Impedance at the fedtru terminal with gap open and shorted.

The feed-thru has the character of a resonant circuit at $47.6 \mathrm{MHz}$ with the parameters derived from the shorted gap impedance to be $Q \approx 9, L \approx 0.36 \mu \mathrm{H}$, and $C \approx 31 \mathrm{pF}$. The circuit capacity is larger than that of the free-standing feed-thru, $\sim 11 \mathrm{pF}$, by its installment into the magnet vessel. The feed-thru brings the resonant frequency of the free-standing magnet from $\sim 32$ down to $\sim 16 \mathrm{MHz}$.

\section{Magnet with $25 \Omega$ termination}

The results for the magnet with, as well as without, feed-thru, but with $25 \Omega$ termination, are shown in Fig. 10 where the wire and direct impedances are compared. The ratio of the resistive components from wire and direct measurements is shown in Fig.11.
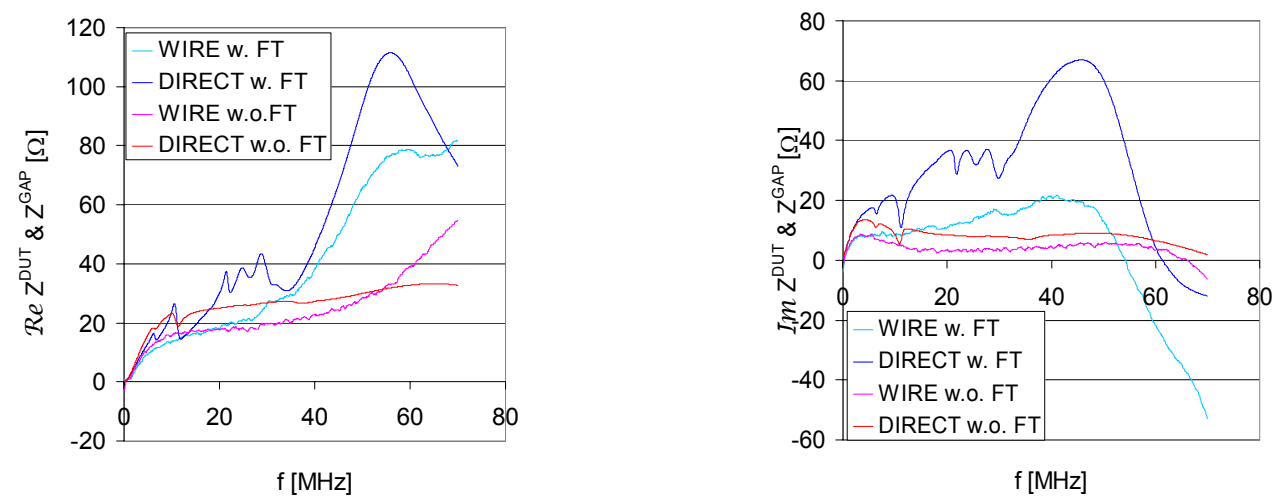

FIG. 10. Impedance with $25 \Omega$ termination from wire and direct measurement.

The two methods agree in general over a broad frequency range, but with the direct measurements giving typically the larger value which in part is due to the fact that the 
propagating wave on the wires couples to the quasi-stationary fields in the magnet according to $\cos ^{2} k \ell$. The agreement of the reactive components is deteriorating with frequency.

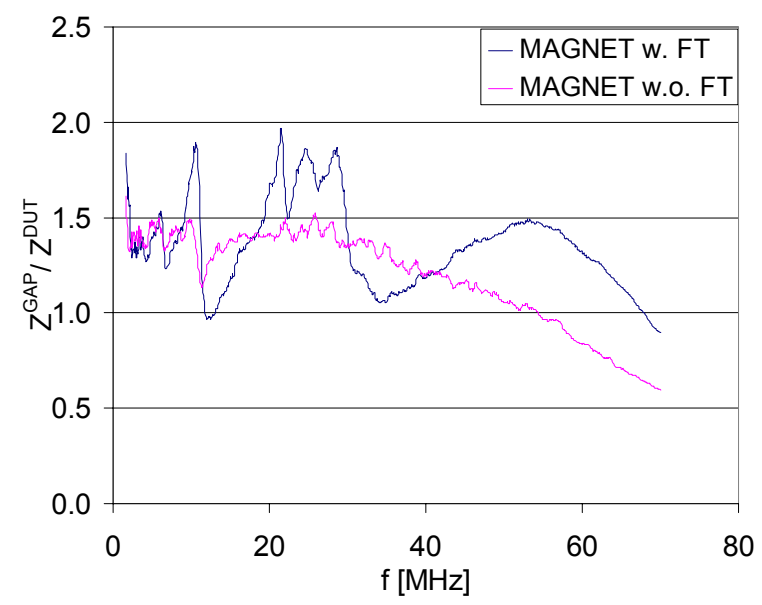

FIG. 11. Ratio of resistive component from wire and direct measurement.

\section{Transverse Coupling impedance of the SNS Extraction Kicker}

The transverse coupling impedance of the SNS extraction kicker with feed-thru and operational $25 \Omega$ termination is shown in Fig.12. The coupled impedance is given with respect to the in-situ reference and the total impedance is estimated by adding the approximation values for the intrinsic impedance. A low-frequency expanded view of the resistive component is shown in Fig. 13.
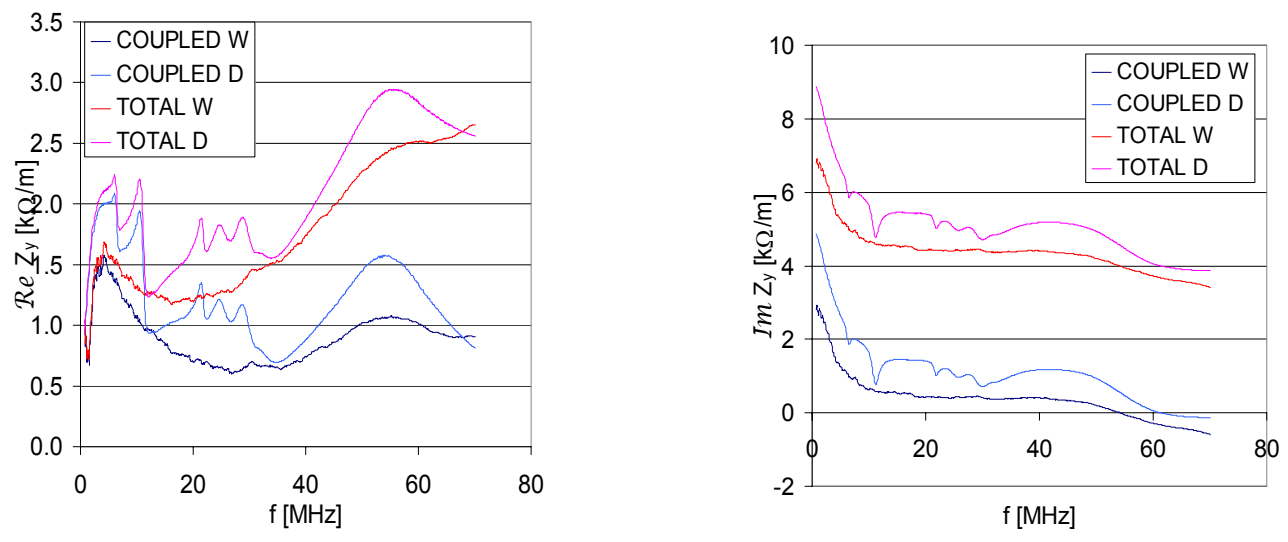

FIG. 12. Total and coupled transverse impedance of the magnet with $25 \Omega$ termination.

The direct measurement has the smallest noise, but the results show that the wire method with the in-situ reference can also give relatively clean signals after application of triple averaging and $1 \%$ smoothing. However, frequencies below $\sim 1 \mathrm{MHz}$ require further study and the graphs are cut off at $600 \mathrm{kHz}$. Extending the measurements to even lower frequencies will require the multi-loop method [9]. 


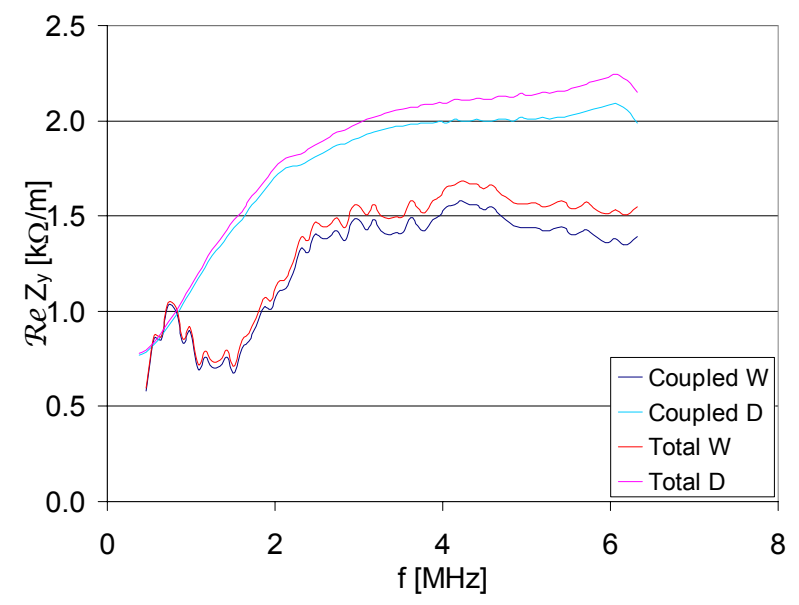

FIG. 13. Transverse Impedance at low frequencies

The measurements from the wire and direct method are compared in these figures and allow some general comments as to their merits. The two measurements show a general agreement of the impedances. The figure with the expanded low-frequency measurements is of particular interest, indicating close agreement between wire and direct measurements at the lowest frequencies. It is noted that at the low frequencies the results from the direct impedance measurement are cleaner and more credible. The agreement is reduced at higher frequencies although a better than qualitative agreement exists.

The direct method is simple to use and requires minimal equipment and set-up time and gives the cleanest signals, even without smoothing, but serves mainly as a lowfrequency method to determine the coupled impedance contribution. The measurement of the intrinsic impedance remains most demanding and is obviously not accessible with the direct method. Furthermore the direct method requires access to the bus-bar gap which is not always possible and is thus restricted to a certain class of kicker magnets. Its proper place is during the design phase to explore options and it is best used in conjunction with the wire method.

\section{Acknowledgements}

It is a pleasure to thank F. Caspers for reading the report. The author acknowledges the help provided by J. Cupolo in performing the TDR measurements on the twin-wire lines. C. Trabocchi and C. Rhein provided essential technical support. 


\section{References}

1. K. Y. Ng, in Handbook of Accelerator Physics and Engineering, edited by A. W. Chao and M. Tigner (World Scientific, Singapore, 1998), p.203

2. R.L.Gluckstern and S. S. Kurennoy, in Handbook of Accelerator Physics and Engineering, edited by A. W. Chao and M. Tigner (World Scientific, Singapore, 1998), p.195

3. T. Weiland, in Handbook of Accelerator Physics and Engineering, edited by A. W. Chao and M. Tigner (World Scientific, Singapore, 1998), p.199

4. F. Caspers, in Handbook of Accelerator Physics and Engineering, edited by A. W. Chao and M. Tigner (World Scientific, Singapore,1998), p.571

5. G. Nassibian and F. Sacherer, Nucl.Instr.\&Methods, 159, 21 (1979)

6. F. Caspers, Proc. 1985 PAC, Vancouver,BC, p. 1914

7. L.S. Walling, D.E. McMurry, D.V. Neuffer and H.A. Thiessen, Nuclear Instruments \& Methods A 281, p. 433-447 (1989).

8. V. G. Vaccaro, INFN-Napoli report, INFN/TC-94/023 (1994); M. Cardito, G. Di Massa, F. Galluccio, R. Losito, M. R. Masullo, V. G. Vaccaro, in Proc. 1993 PAC, Washington, DC, p. 2154

9. F. Caspers, A. Mostacci, L. Vos, informal report LCC160102.pdf, (private communication)

10. D. Davino and H. Hahn, Phys. Rev. ST, Accelerators and Beams, 6, 012001 (2003)

11. M. M. Blaskiewicz, private communication.

12. U. Blell, in Proc. 1999 PAC, New York, NY, p. 1727

13. F. Voelker and G. Lambertson, Proc. PAC 1989, Chicago, IL, p. 851

14. S. Y. Zhang, BNL Report, C-AD/AP/20 (2000)

15. S. Ramo, J. R. Whinnery, Th. Van Duzer, Fields and Waves in Communication Electronics, (John Wiley \& Sons, New York,1994) 3. ed., p.250.

16. H. Hahn and F. Pedersen, BNL 50870 (1978)

\section{Appendix}

Formal expressions for the forward scattering coefficient associated with a series impedance, $Z$, between twin-wire lines are derived here from elementary circuit theory without the explicit use of hybrid matrices. The notation used is exhibited in Fig. A1.

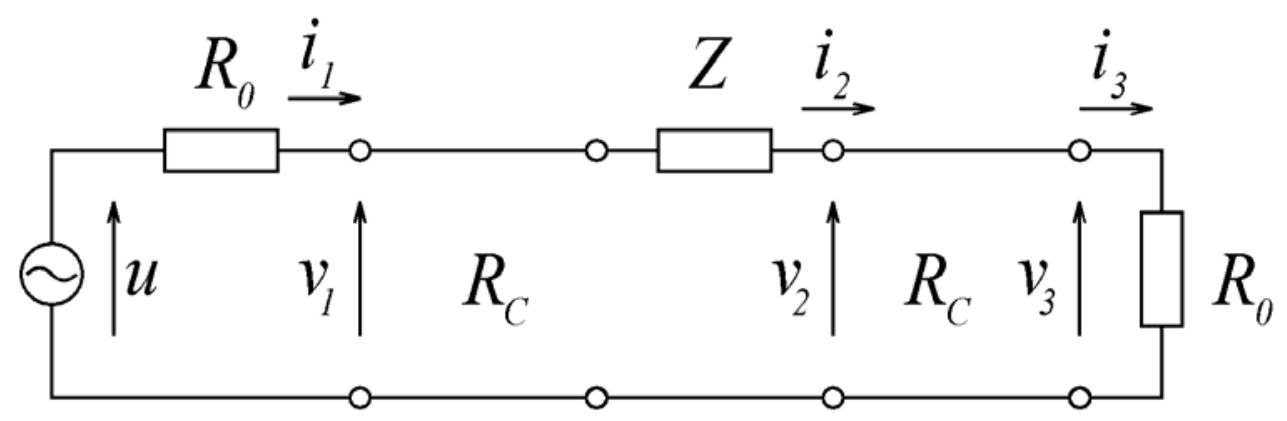

Fig. A1. Circuit model for twin-wire impedance measurements. 
The forward scattering coefficient is defined as

$$
S_{21}=2 v_{3} / u
$$

and is obtained by sequential elimination of the currents and voltages in the following equations:

$$
\begin{aligned}
& v_{3}=v_{2} \cos k \ell-j R_{C} i_{2} \sin k \ell \\
& v_{2}=\frac{Z_{2}}{Z_{2}+Z}\left(v_{1} \cos k \ell-j R_{C} i_{1} \sin k \ell\right) ; i_{2}=i_{1} \cos k \ell-j R_{C}^{-1} v_{1} \sin k \ell \\
& v_{1}=\frac{Z_{1}}{R_{0}+Z_{1}} u ; i_{1}=\frac{1}{R_{0}+Z_{1}} u, \\
& Z_{1}=\frac{Z+Z_{2}+j R_{C} \tan k \ell}{1+\left(Z+Z_{2}\right) R_{C}^{-1} \tan k \ell} ; Z_{2}=\frac{R_{0}+j R_{C} \tan k \ell}{1+R_{0} R_{C}^{-1} \tan k \ell} .
\end{aligned}
$$

The complete expression for the scattering coefficient is obtained via the MAXIMA program but is too large for presentation here. The Taylor expansion for low frequencies, $k=\omega / c$, follows as

$$
S_{21}=\frac{2 R_{0}}{2 R_{0}+Z}-j 4 k \ell \frac{R_{0}\left(R_{0}^{2}+R_{C}^{2}+R_{0} Z\right)}{R_{c}\left(4 R_{0}^{2}+4 R_{0} Z+Z^{2}\right)}
$$

The coefficient ratio for the interpretation of the measurements is now

$$
\frac{S_{21}^{D U T}}{S_{21}^{R E F}} \approx \frac{2 R_{0}}{2 R_{0}+Z}+j 2 k \ell \frac{Z}{R_{C}} \frac{R_{C}^{2}-R_{0}^{2}}{4 R_{0}^{2}+4 R_{0} Z+Z^{2}}
$$

(A7)

The scattering coefficient ratio for a parallel conductance, $Y$, is found to be

$$
\frac{S_{21}^{D U T}}{S_{21}^{R E F}} \approx \frac{2}{2+R_{0} Y}-j 2 k \ell Y R_{C}^{-1} \frac{R_{C}^{2}-R_{0}^{2}}{4+4 Y R_{0}+Y^{2} R_{0}^{2}} .
$$

